\title{
UVCS/SOHO investigation of the interface between streamers and coronal holes ${ }^{\star}$
}

\author{
D. Spadaro ${ }^{1}$, R. Ventura ${ }^{1}$, G. Cimino ${ }^{1}$, and M. Romoli ${ }^{2}$ \\ 1 INAF - Osservatorio Astrofisico di Catania, via S. Sofia 78, 95123 Catania, Italy \\ e-mail: dspadaro@ct.astro.it \\ 2 Dipartimento di Astronomia e Scienza dello Spazio, Università di Firenze, L.go E. Fermi 2, 50125 Firenze, Italy
}

Received 23 February 2004 / Accepted 30 August 2004

\begin{abstract}
We investigated the properties of the interface between streamers and coronal holes at low heliocentric distances, observing the extended solar corona in the North-West quadrant by UVCS/SOHO. We measured the line profiles of the H I Ly $\alpha$ and O VI resonance doublet and the visible linearly polarized radiance at heliocentric distances ranging from 1.4 to $2.5 R_{\odot}$, and colatitudes spanning from the North pole to the West equator with steps of $\sim 10^{\circ}$. The results show that both the line intensities and the line widths, in particular those of O VI, exhibit sharp variations across the streamer boundaries, with a clear anticorrelation between intensities and widths. We also notice a positive correlation for Ly $\alpha$ in the region close to the equator. The steep changes in O VI line profiles occur in a narrow transition region $\left(5^{\circ}-10^{\circ}\right)$, right at the borders of the streamers, from $1.5 R_{\odot}$ onwards. The $\mathrm{O}$ VI resonance doublet line ratio steeply increases outside of the streamer as well, but this occurs at higher heliocentric distances (above $2 R_{\odot}$ ). Hence the marked broadening of the $\mathrm{O}$ VI lines and the considerable rise of their intensity ratio are an evident signature of the transition from closed to open field lines in streamer magnetic field topologies. This behaviour also implies that a strong and preferential non-thermal heating of O VI ions in the direction coinciding with the line of sight and the turn-on of a significant outflow occur in the open magnetic field region near or just outside of the streamer edges.
\end{abstract}

Key words. Sun: corona - Sun: solar wind - Sun: UV radiation

\section{Introduction}

The competition between solar wind expansion and plasma trapping by closed bipolar magnetic fields, caused by the high thermal and electrical conductivities of the corona, gives rise to two typical states in the extended solar atmosphere: the magnetically open coronal holes and the magnetically closed helmet streamers (see, e.g., Low 2001).

Coronal holes appear as extended darker regions with a density significantly lower (by a factor of three) than the typical background corona, and probably with a lower temperature too (see, e.g., Priest 1987). They possess an open diverging magnetic structure and lie above some of the more extensive unipolar regions in the photosphere. Near the solar activity minimum long-lasting coronal holes are present above both poles of the Sun.

The large-scale closed structures surmounted by a blade of open fields and known as coronal streamers contain a high pressure, high density plasma because the closed magnetic field is strong enough to confine it. At about $1-2 R_{\odot}$ above the solar surface, magnetic and plasma body forces are comparable, beyond which all magnetic lines of force are kept open by the expanding solar wind and form a neutral current sheet

* Figures 2, 4-6 and 8-10 are only available in electronic form at http://www.edpsciences.org
(Priest 1987). Hence streamers appear as elongated structures protruding from the Sun towards interplanetary space up to $10 R_{\odot}$ (Noci et al. 1997). During solar maximum they can be found at almost every latitude, while during minimum they are located around the solar equator, forming the so-called streamer belt.

This evident dichotomy of two states in the extended solar corona can be related to the bimodal character of the solar wind detected by the Ulysses spacecraft (Phillips et al. 1995): either fast $\left(750-800 \mathrm{~km} \mathrm{~s}^{-1}\right)$ or slow $\left(\sim 400 \mathrm{~km} \mathrm{~s}^{-1}\right)$ flow speeds have been measured at $1 \mathrm{AU}$, with few values in between. In fact, it is generally agreed upon that coronal holes are the main source regions of the fast, mostly uniform, solar wind (e.g., Priest 1987; Marsch 1997; Cranmer et al. 1999). Conversely, the slow solar wind, which shows large excursions in speed and elemental abundances, is believed to originate in streamers (e.g., Marsch 1997; Noci et al. 1997; Raymond et al. 1997), even if a firm identification of the source regions is still under investigation.

Some authors (e.g., Habbal et al. 1997; Strachan et al. 2002), for instance, have found evidence of low velocity outflows from regions along the axis of streamers just above the closed field lines structure, also known as stalks. Others (e.g., Noci et al. 1997; Raymond et al. 1997) have pointed out that the legs of streamers, i.e. the regions close to their boundaries, 
exhibit the same abundance anomalies with respect to the photosphere as the slow solar wind, so that these legs might be the real source regions of the slow wind. It is worth noting that some theoretical models also identify the regions of open magnetic field lines near the streamer-coronal hole boundaries as the source of the slow solar wind (e.g., Wang 1994; Ofman 2000).

All this stimulates investigations on the detailed properties of the interface between streamers and coronal holes at low heliocentric distances (i.e., below $5 R_{\odot}$ ) and, in particular, studies on the variation of the physical conditions of the coronal plasma across the streamer boundaries. This can be accomplished, for instance, by the UVCS spectrocoronagraph (Kohl et al. 1995) on board the SOHO spacecraft (Domingo et al. 1995), studying the basic properties of some line profiles (e.g., the H I Ly $\alpha$ and O VI resonance doublet) and of the linearly polarized visible radiance as a function of heliocentric distance and latitude. We refer the reader to Kohl et al. (1995) for a comprehensive description of the UVCS experiment.

The latitudinal behaviour at low heliocentric distances of the emission line intensities and widths has been recently investigated by some authors using UVCS/SOHO data collected when the Sun was close to the minimum of its activity (Antonucci et al. 1997; Dobrzycka et al. 1999; Strachan et al. 2000; Zangrilli et al. 1999, 2002). They mainly focused on the differences between the only two types of large scale structures present: extended polar coronal holes and equatorial streamers. Strachan et al. (2002), moreover, studied the variations of $\operatorname{Ly} \alpha$ and $\mathrm{O}$ VI line profiles as a function of latitude at $\sim 2.3 R_{\odot}$ from Sun centre in an equatorial streamer at solar minimum. They selected only two slit orientations, covering a latitude range of $\pm 25^{\circ}$ from the streamer axis, and found a relatively sharp transition from regions of no measurable outflow within the streamer core to positive outflows just outside of the streamer legs.

To carry out a more extensive research into the properties of the interface between streamers and coronal holes, we observed the extended corona close to the solar limb in the North-West quadrant by UVCS/SOHO throughout a week during the second MEDOC (Multi-Experiment Data Operations Centre for SOHO) campaign (October-November 1997), coordinated at the Institut d'Astrophysique Spatiale in Orsay - Paris. The line profiles and visible polarized radiance were measured at several heliocentric distances, in the interval from 1.4 to $2.5 R_{\odot}$, and for colatitudes ranging from the pole to the equator with steps of $\sim 10^{\circ}$, in order to study in high detail their dependence on radial distance and latitudinal angle all over the NW quadrant. This paper reports on the results obtained from the analysis of such spectroscopic data, carried out with the main purpose of deeply investigating the variations of wind parameters across the streamer boundaries and providing a further and more detailed input for theoretical models of streamers and the source regions of the slow solar wind. It is remarkable, in this respect, that a high-latitude streamer $\left(\sim 50^{\circ}\right.$ above the equatorial plane) was also present in the quadrant selected for the observations (see Figs. 7-10), besides another one close to the equatorial belt, hence increasing the number of structures in the data set suitable for our investigation.
Table 1. Log of the observations.

\begin{tabular}{ccl}
\hline \hline Date & Hel. dist. $\left(R_{\odot}\right)$ & Selected PA $\left(^{\circ}\right)$ \\
\hline Oct. 27 & 1.39 & $270,280,290,300,310,330,360$ \\
Oct. 28 & 1.51 & $270,280,290,300,310,330,360$ \\
Oct. 29 & 1.63 & $270,280,290,300,310,330$ \\
Oct. 30 & 1.75 & $270,280,290,300,310,330$ \\
Oct. 31 & 1.98 & $270,280,290,300,310$ \\
Nov. 1 & 2.21 & $270,280,290,300$ \\
Nov. 2 & 2.55 & $270,280,295$ \\
\hline
\end{tabular}

Note. The nominal heliocentric distance and PA for each slit refer to the distance and PA of the point along the slit that is closest to the limb. This reference is shown by the white dot on each slit in Figs. 7-10.

\section{Observations and data analysis}

The data on which this work is based have been acquired during the week from October 27 to November 2, 1997. They consist of intensities and profiles of $\operatorname{Ly} \alpha$ and O VI resonance doublet (1032 $\AA, 1037 \AA$ ) lines, and of linearly polarized visible radiance, simultaneously measured at a given heliocentric distance for colatitudes ranging from the pole to the equator in the North-West quadrant of the extended solar corona.

The selected heliocentric distances (one per day) range from $\sim 1.4$ to $\sim 2.5 R_{\odot}$ and are reported in Table 1 . The table also reports the position angles (PAs, measured counterclockwise from the solar North pole), i.e. the orientations of the UVCS entrance slits for each selected heliocentric distance. PA ranges from $270^{\circ}$ to $360^{\circ}$ in the NW quadrant. Note that for the largest heliocentric distances we considered a smaller set of position angles, owing to the weaker and weaker emission of the solar corona and the consequent need for longer exposure times to get satisfactory signal to noise ratios. For this reason we also discarded slit orientations close to the North pole.

The spatial coverage along the slit of the Ly $\alpha$ and $\mathrm{O}$ VI channels is $\sim 40$ arcmin, while the visible light channel observes only a region of $14 \operatorname{arcsec} \times 14$ arcsec about the centre of the slits. Spatial information has been binned over 6 pixels, each bin corresponding to 42 arcsec. The selected Ly $\alpha$ entrance slit width is $50 \mu \mathrm{m}$, while for the O VI channel it was varied from $50 \mu \mathrm{m}$ to $200 \mu \mathrm{m}$, depending on the heliocentric distance and PA, in order to optimize both spectral resolution and photon flux.

We used the most recent version of the UVCS Data Analysis Software (DAS) to remove the image distortion, to flat-field the detectors, to perform the wavelength and radiometric calibration of the data and to extract the spectra (Gardner et al. 1996; Kohl et al. 1997). We subtracted the contributions from the disc scattered stray light affecting both the $\mathrm{HI} \operatorname{Ly} \alpha$ and $\mathrm{O}$ VI lines, as well as the additional contribution from interplanetary scattered $\operatorname{Ly} \alpha$ contained in the observed Ly $\alpha$ profile, according to the standard technique described by Kohl et al. (1997). The resulting profiles of Ly $\alpha$ and $\mathrm{O}$ VI resonance doublet lines, after the removal of the instrumental broadening and backgrounds (see Kohl et al. 1997), were curve-fitted to single Gaussians in order to determine intensity values and $1 / e$ half widths for these lines. The $1 / e$ half widths, translated into Doppler velocity widths, provide direct 
measurements of the velocity distribution of particles along the line-of-sight (see, e.g., Cranmer et al. 1999), related to the kinetic temperature of the plasma. We also determined the linearly polarized radiance $(p B)$ of the corona in the wavelength band from $4500 \AA$ to $6000 \AA$ according to the method reported in Romoli et al. (2002). We describe the outcome of this analysis in the following section.

\section{Results and discussion}

Figures 1 and 2 show the latitudinal distribution of the O VI $1032 \AA$ and H I Ly $\alpha$ total intensities (integrated over the profile), respectively, at the different heliocentric distances considered during the week of observations (see Table 1). The sample intervals of the latitudinal scans reported in the figures are $5^{\circ}$. This is accomplished by taking several spatial points along the slit for each orientation; they correspond to different heliocentric distances and hence determine the height ranges characterizing the various panels in the figures. For a given latitudinal scan, moreover, we averaged the intensities obtained for different slit orientations in points corresponding to the same PA, and reported the resulting values in the relevant plot.

The uncertainties in the line intensities are mainly due to radiometric calibration, stray light correction and photon counting statistics (Dobrzycka et al. 1999; Gardner et al. 2002; Strachan et al. 2002), even if only the last source (1-2\%) is important for the purposes of our investigation, whereas the others should affect measurements in a systematic way, in case shifting all data by about $10 \%$ (see also, Zangrilli et al. 1999). Hence the error bars $( \pm 1 \sigma)$ are based on the counting statistics; they are not plotted in Figs. 1 and 2 because smaller than the symbol size.

The plotted line intensities clearly exhibit the signatures of the two streamers appearing in the NW quadrant (see also Figs. 7-10), whose level of ultraviolet emission is significantly higher than that in the polar coronal hole (about one order of magnitude) and in the inter-streamer regions (at least a factor of two). Such differences are better distinguishable for the O VI $1032 \AA$ intensity; therefore we identified the angles corresponding to the axes of the examined streamers as the positions of the O VI line intensity maxima. Then, for each streamer, we defined its borders as the angles corresponding to intensity values equal to $1 / e$ the relevant maximum. According to this defintion, the lower PA border of the high latitude, fainter streamer can result embedded in the tail of emission due to the brighter streamer closer to the equator, particularly at heliocentric distances below $1.75 R_{\odot}$. In this case we set the border in the position of minimum $\mathrm{O}$ VI intensity between the axes (see Fig. 1).

It is worth noting the narrow transition region $\left(\sim 10^{\circ}\right)$ at which the line intensities sharply increase from the low polar coronal hole level, rather constant over a range of about $40^{\circ}$ in latitude, to the much higher level corresponding to the streamer at $50^{\circ}-60^{\circ}$ above the equatorial plane (see panels a-d, relevant to a range of heliocentric distances from $\sim 1.4$ to $\sim 1.9 R_{\odot}$ ). This steep increase is similar to that found by, e.g., Dobrzycka et al. (1999) and Strachan et al. (2002) for streamers in the equatorial belt. A considerable variation in the line intensities (more than one order of magnitude) over about $20^{\circ}$ (from

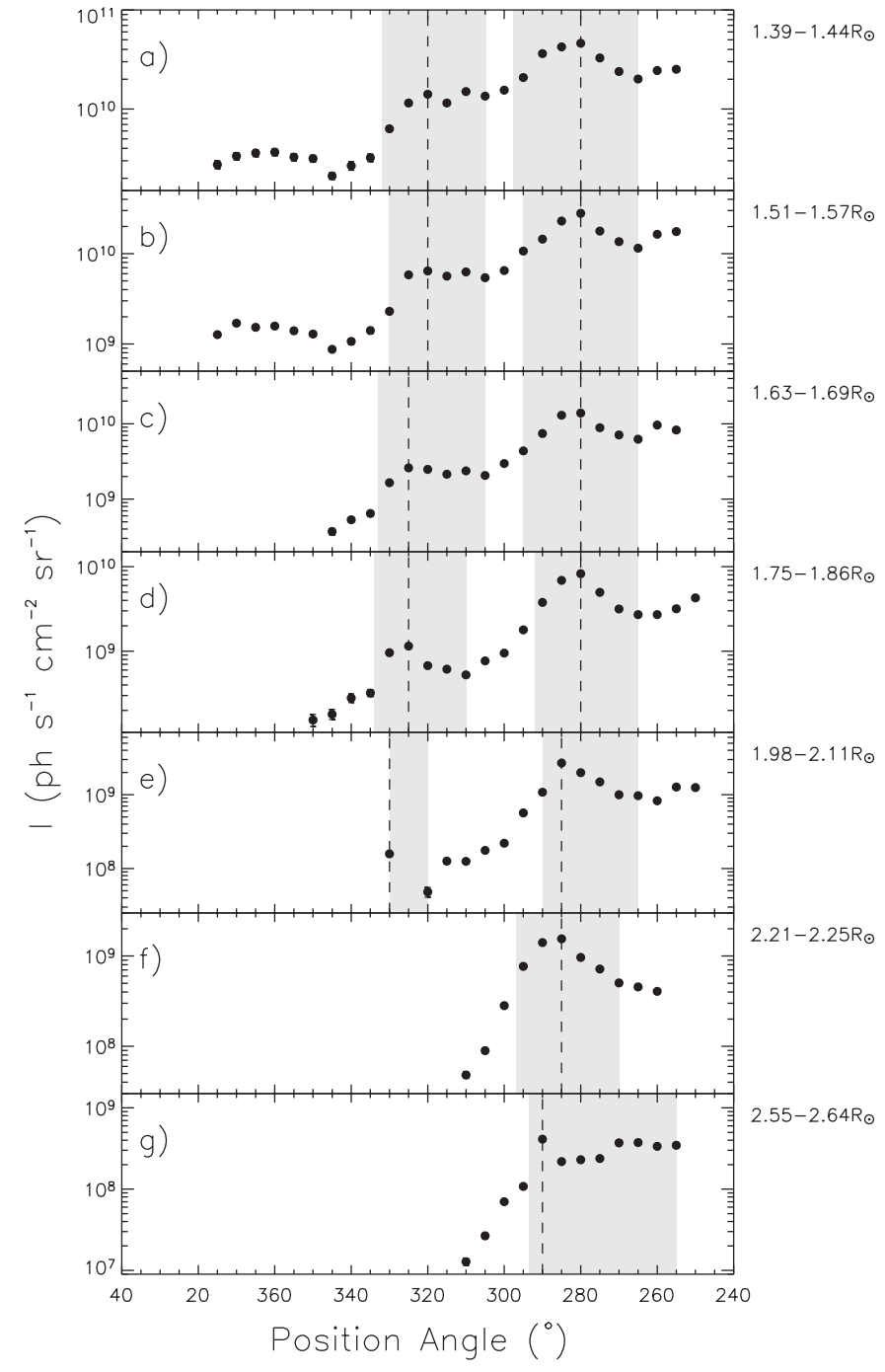

Fig. 1. Intensity of the O VI $1032 \AA$ spectral line vs. position angle, measured counterclockwise from the solar North pole, for the heliocentric distance ranges indicated on the right of each panel. The vertical dashed lines mark the positions of the axes of the streamers, identified as the maxima of the $\mathrm{O}$ VI line intensity. The grey bands represent the extent in PA of the streamers; their bounds are defined by the $1 / e$ intensity values of the $\mathrm{O}$ VI line and indicate the streamer borders.

$\mathrm{PA}=310^{\circ}$ to $\left.\mathrm{PA}=290^{\circ}\right)$ is also noticed for the streamer closer to the equator (at latitudes $\sim 10^{\circ}-20^{\circ}$ ), at heliocentric distances higher than $2.0 R_{\odot}$ (see panels e-g). Moreover, we note that the O VI $1032 \AA$ emission does not exhibit any significant reduction in correspondence of the axis for both streamers appearing in the NW quadrant, contrary to the typical behaviour of streamers in the equatorial belt described by Noci et al. (1997); Raymond et al. (1997); Dobrzycka et al. (1999); Zangrilli et al. (1999); Strachan et al. (2002); Frazin et al. (2003). Our result is confirmed by the observations of Ventura et al. (2004), carried out during the subsequent week of the second MEDOC campaign. These authors, as well as Bemporad et al. (2003), also found no axis reduction of the O VI intensity for streamers outside the equatorial belt observed in 2000, near the maximum of solar activity. 


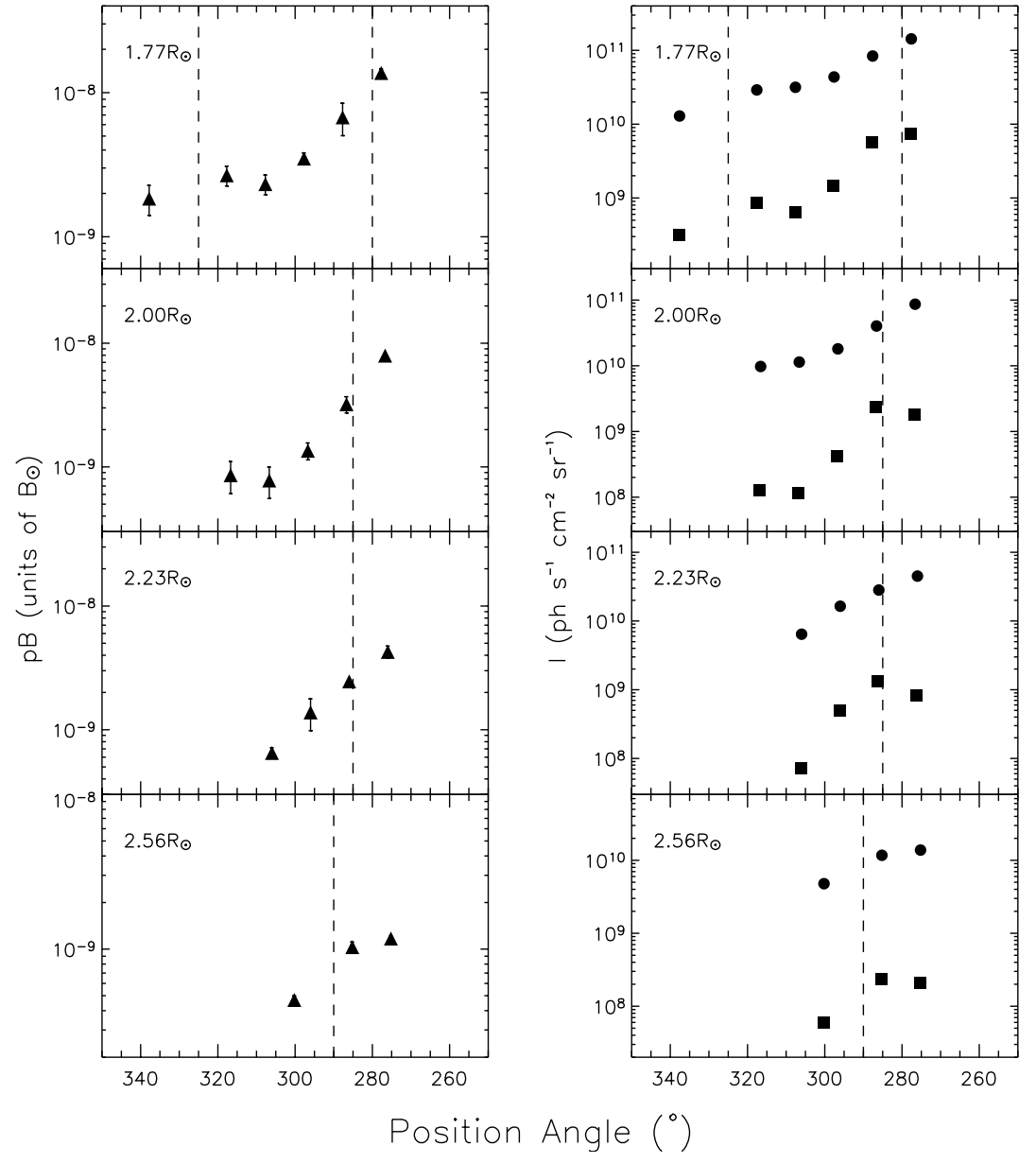

Fig. 3. Left: linearly polarized visible radiance $(p B)$ vs. position angle for the heliocentric distances indicated in each panel. Right: co-registered Ly $\alpha$ (circles) and $1032 \AA$ A (squares) line intensities vs. position angle. The vertical dashed lines mark the same positions of the axes of the streamers identified in Fig. 1 according to the behaviour of the O VI line intensity.
Figure 3 reports the linearly polarized visible radiance $(p B)$, measured in units of the photospheric photon radiance at Sun centre $B_{\odot}$ (Romoli et al. 2002), as a function of position angle for some of the heliocentric distances considered during the week of observations. Slit heights below $1.75 R_{\odot}$ are neglected, because there the stray light level in the visible light channel is too high for a meaningful determination of $p B$ (see, Romoli et al. 2002). The sampling of the latitudinal scans is poorer than in Figs. 1 and 2, only a $14 \operatorname{arcsec} \times 14 \operatorname{arcsec}$ region being observed in the visible for each slit orientation. The error bars are based on the photon counting statistics. The Ly $\alpha$ and $1032 \AA$ total line intensities obtained in the spatial bin along the slit corresponding to the visible light channel pinhole are also reported in Fig. 3.

The $p B$ value is proportional to the integral of the electron density along the line of sight (e.g., Billings 1966). In fact, its latitudinal profile at $1.77 R_{\odot}$ distinctly shows the signatures of both streamers, denser than the other regions in the NW quadrant. Moreover, the $p B$ for the high-latitude structure is considerably lower than that obtained for the structure closer to the equatorial belt. The latitudinal plots relevant to the other heliocentric distances exhibit a firm increase in $p B$ from the less dense inter-streamer regions to the streamer near the equator. It is worth noting that the intensity of the Ly $\alpha$ line, whose emission is also proportional to the integral of the electron density along the line of sight (Gabriel 1971), shows the same dependence on PA as the $p B$. As for the $1032 \AA$ line, we note a similar trend, even if the streamer signatures are slightly more pronounced, due to the enhanced contribution of the collisional component of the line, proportional to the squared electron density (Noci et al. 1987).

Figures 4 and 5 present the latitudinal dependence of the $1 / e$ half widths of O VI $1032 \AA$ and H I Ly $\alpha$ profiles, respectively, at the same heliocentric distances reported in Figs. 1 and 2 . The uncertainties affecting these line widths have been evaluated by the same procedure adopted by Zangrilli et al. (1999). The corresponding $\sigma$ values are shown as error bars in the figures.

We note that the $1 / e$ half widths of the O VI line measured in the two streamers are significantly narrower than in the other regions of the NW quadrant, in particular the polar coronal hole, where the profiles can be up to a factor of 5 broader (see panels a-d in Fig. 4), while the H I Ly $\alpha$ line widths appear to be relatively constant with latitude, only increasing by $20-30 \%$ in the polar coronal hole (see panels a-d in Fig. 5). It is interesting that even the high-latitude narrow and faint streamer causes an abrupt decrease in the $\mathrm{O}$ VI line width (by at least a factor of 3) with respect to both the polar coronal hole (Fig. 4d) and the inter-streamer region (Fig. 4e). The region between streamers also exhibits broader O VI profiles than in the streamers, especially at heliocentric distances above $1.9 R_{\odot}$ (see panels e-g in Fig. 4). As for the Ly $\alpha$ line, conversely, we note slightly wider $(\sim 10 \%)$ profiles inside the streamer regions than in the interstreamer one. This is more evident for the streamer closer to 
Table 2. Correlation of variations in line intensities and widths.

\begin{tabular}{ccc}
\hline \hline & O VI 1032 А & H I Ly $\alpha$ \\
\hline Hel. dist. $\left(R_{\odot}\right)$ & Rank correl. coeff. & Rank correl. coeff. \\
\hline $1.39-1.44$ & $-0.89\left(2.1 \times 10^{-9}\right)$ & $-0.84\left(1.4 \times 10^{-7}\right)$ \\
$1.51-1.57$ & $-0.92\left(1.2 \times 10^{-9}\right)$ & $-0.35\left(8.4 \times 10^{-2}\right)$ \\
$1.63-1.69$ & $-0.88\left(6.5 \times 10^{-7}\right)$ & $-0.05\left(8.4 \times 10^{-1}\right)$ \\
$1.75-1.86$ & $-0.89\left(9.2 \times 10^{-8}\right)$ & $0.15\left(5.1 \times 10^{-1}\right)$ \\
$1.98-2.11$ & $-0.79\left(2.8 \times 10^{-4}\right)$ & $0.37\left(1.4 \times 10^{-1}\right)$ \\
$2.21-2.25$ & $-0.36\left(2.7 \times 10^{-1}\right)$ & $0.62\left(4.3 \times 10^{-2}\right)$ \\
$2.55-2.64$ & $-0.85\left(4.2 \times 10^{-4}\right)$ & $0.80\left(1.9 \times 10^{-3}\right)$ \\
\hline
\end{tabular}

Note. The significance value for each rank correlation coefficient is reported in brackets. The whole latitudinal intervals are considered.

the equator, at heliocentric distances above $1.7 R_{\odot}$. Such behaviour is similar to that described by Zangrilli et al. (1999), who found that in the $1.5-2.0 R_{\odot}$ range the Ly $\alpha$ width is broader inside the equatorial belt streamer, decreases with latitude outside it to about $50-60^{\circ}$ above the equatorial plane and then increases towards the pole.

Analogously to the line intensities, the O VI $1032 \AA 1 / e$ half widths sharply change over a narrow transition region $\left(\sim 10^{\circ}\right)$, exhibiting a marked broadening of the line profiles right at the boundaries of the streamers. The steep increases in the line width already occur at low heliocentric distances across the border between the high latitude streamer and the polar coronal hole (by about a factor of 2 below $1.7 R_{\odot}$ and by more than a factor of 3 around $1.8 R_{\odot}$ ). A similar result was obtained by Antonucci et al. (1997), whereas other authors (e.g., Dobrzycka et al. 1999; Zangrilli et al. 1999) found considerable latitudinal variations in the line widths only above $2.0 R_{\odot}$.

The steep variations in the O VI line width and intensity clearly appear anti-correlated (cf. Figs. 1 and 4), as previously discussed by Antonucci et al. (1997) and Abbo \& Antonucci $(2002 a, b)$, confirming that the width of the ion velocity distribution along the line-of-sight increase dramatically outside the streamers, where open magnetic field lines are expected to predominate. In order to make a quantitative analysis of this correlation, we calculated Spearman's rank correlation coefficients (Press et al. 1986) for the whole latitudinal intervals examined at the various heliocentric distances. The adopted procedure also gives the two-sided significance of the deviation from zero of the coefficient. The significance is a value in the interval $[0.0,1.0]$; a small value indicates a significant correlation. The rank correlation coefficients, with the relevant significances, calculated for the O VI $1032 \AA$ and H I Ly $\alpha$ lines from the data plotted in Figs. 1 and 4 and in Figs. 2 and 5, respectively, are reported in Table 2 . These results confirm the significant anti-correlation between the variations in the O VI line width and intensity all over the latitudinal intervals considered, whereas for the Ly $\alpha$ line the correlation is generally poorer and changes its sign, becoming positive towards higher heliocentric distances.

Given the high latitudinal resolution of the observations, it is possible to analyse the correlation between variations in line width and intensity selecting some specific PA ranges, so as to investigate the two streamer regions separately. For instance, if we examine the high latitude streamer together with the adjacent polar coronal hole $\left(\mathrm{PA} \geq 300^{\circ}\right.$ ) for the various height ranges, we get a rank correlation coefficient for $\mathrm{O}$ VI between -0.82 and -0.91 , with a significance of 0.0001 at most. As for Ly $\alpha$, the coefficient ranges from -0.57 to -0.91 , with a significance always below 0.02 . These coefficients considerably increase when the analysis concentrates on the PA range crossing the Northern border of the streamer $\left(325^{\circ}-350^{\circ}\right)$ : in the 1.75-1.86 $R_{\odot}$ height range (see panel d in Figs. 1, 2, 4 and 5), we get -1.0 and -0.95 , with relevant significance 0.0 and 0.0048 , for O VI and $\operatorname{Ly} \alpha$, respectively. That is practically a linear anti-correlation between the variations in line width and intensity across this sharp boundary. On the other hand, considering the region closer to the equatorial belt $\left(260^{\circ} \leq \mathrm{PA} \leq\right.$ $300^{\circ}$ ), the rank correlation coefficient for $\mathrm{O}$ VI is -0.1 at most, with a significance always about 0.9 , while in the case of Ly $\alpha$ we get positive coefficients between 0.6 and 0.8 and a significance smaller than 0.05 . This result can explain the behaviour with the heliocentric distance of the Ly $\alpha$ coefficients reported in Table 2, also taking into account that the latitudinal scans above $2.0 R_{\odot}$ discard the slit orientations close to the North pole and hence enhance the contribution of the equatorial region to the resulting rank correlation parameters. It is also in agreement with the slight increase in Ly $\alpha$ width noticed inside streamers close to the equator (see the discussion above and, also, Zangrilli et al. 1999).

Therefore, the analysis of the correlation between variations in line intensity and broadening carried out in different PA intervals of the NW quadrant points out a different behaviour in the high latitude streamer region with respect to that closer to the equator. Here, in particular, the two examined lines also exhibit significantly different correlation characteristics. However, the variations across the sharp boundary between the streamer and the polar region are strongly anti-correlated for both lines.

The ratio of the intensities of the O VI resonance doublet lines can be used as a probe of the wind outflow velocity in the extended solar corona, adopting the Doppler dimming technique developed by the UVCS team (Noci et al. 1987; Dodero et al. 1998; Li et al. 1998; Cranmer et al. 1999). Figure 6 reports the O VI line ratio $\left(I_{1037} / I_{1032}\right)$ as a function of position angle for the various heliocentric distances considered during the week of observations. The uncertainties in the line ratios have been deduced from the statistical uncertainties in the relevant line intensities. The positions of the axes and borders of the streamers, defined according to the behaviour of the O VI $1032 \AA$ line intensity, are marked in the figure.

The line ratio appears to be relatively constant with latitude for all heliocentric distances up to $2.1 R_{\odot}$. The values decrease from $\sim 0.4$ to $\sim 0.3$ with increasing heliocentric distances, as a consequence of the radial decrease in plasma density which makes the resonantly scattered component of the O VI lines to contribute to the line intensities significantly more than the collisionally excited one. Note that for purely collisionally excited lines the ratio is $\simeq 0.5$, whereas for lines excited only by resonant scattering of the solar disc radiation the ratio is close to 0.25 (e.g., Noci et al. 1987). We hint from these values 
that the outflow plasma velocity cannot exceed $\sim 50 \mathrm{~km} \mathrm{~s}^{-1}$ (cf. Noci et al. 1987; Dodero et al. 1998), especially in the centre of the streamers. It is worth noting that Strachan et al. (2002) also deduced outflow velocities $\lesssim 50 \mathrm{~km} \mathrm{~s}^{-1}$ up to $4 R_{\odot}$ along the axis of an equatorial streamer. On the other hand, Ventura et al. (2004) measured O VI line ratios $\sim 0.3$ up to $3.8 R_{\odot}$ inside streamer structures as well.

Above $2.2 R_{\odot}$, however, the O VI intensity line ratio abruptly increases from $\sim 0.3$ to $\sim 0.5$, in a $5^{\circ}-10^{\circ}$ latitude interval outside of the equatorial streamer structure (see panels $\mathrm{f}$ and $g$ in Fig. 6). According to Noci et al. (1987) and Dodero et al. (1998), we deduce an increase of the plasma outflow velocity from values below $50 \mathrm{~km} \mathrm{~s}^{-1}$ within the streamer core and at its borders to $\sim 100 \mathrm{~km} \mathrm{~s}^{-1}$ at $10^{\circ}-15^{\circ}$ above the Northern boundary of the streamer, where the Doppler dimming effects significantly reduce the resonantly scattered component of the OVI lines and consequently make the intensity ratio rise. This is consistent with the $\mathrm{O}$ VI outflow velocities determined by Strachan et al. (2002) as function of latitude at $2.3 R_{\odot}$ from Sun centre in an equatorial streamer. Moreover, the differences in PA between the streamer boundary, identified by the $1 / e \mathrm{O}$ VI intensity value, and the angle where the line ratio is $\sim 0.5$ are similar to the analogous values $\left(10^{\circ}-20^{\circ}\right)$ obtained by Abbo \& Antonucci (2002a) in the 2.1-2.6 $R_{\odot}$ range of heliocentric distance, assuming the same definition for the actual streamer border.

It is also interesting a comparison with the measurements of the OVI line ratio in the extended solar corona previously discussed by Antonucci et al. (1997) and Habbal et al. (1997). Both investigations obtained the 0.5 value of the ratio, revealing outflow velocities $\sim 100 \mathrm{~km} \mathrm{~s}^{-1}$, well above $4.0 R_{\odot}$ along the axis of the examined streamers (note that our observations do not cover this range of heliocentric distance). Outside of the streamers, in mid-latitude regions, Antonucci et al. (1997) found a line ratio $\sim 0.5$ at position angles differing by at least $30^{\circ}$ from the axis of the structure below $2.9 R_{\odot}$, whereas Habbal et al. (1997) got this value at PA $\pm 10^{\circ}$ from the axis, in the 3.5-4.0 $R_{\odot}$ height range, and at $\mathrm{PA} \pm 20^{\circ}$, in the 2.5-3.0 $R_{\odot}$ range. The observations discussed in the present paper give a value $\sim 0.5$ for the $\mathrm{O}$ VI line ratio at $\sim 25^{\circ}$ from the streamer axis around $2.2 R_{\odot}$, and at $\sim 15^{\circ}$ around $2.6 R_{\odot}$.

Figures 7-10 report the values along the slit, for some selected positions, of the Ly $\alpha$ and O VI $1032 \AA$ total line intensity and $1 / e$ half width, as well as of the $\mathrm{O}$ VI line ratio, to illustrate their variations across the streamer boundaries in more detail. As for the line ratio, the values plotted for all PAs but those at slit ends result from a running mean, calculated averaging over three nearby values. This reduces the error bars of the ratios, although at the expenses of the latitudinal resolution that can be used for identifying the transition in this parameter. The figures also show the configuration of the extended solar corona in the LASCO C1 and C2 visible light images obtained during the relevant days of observation. We refer the reader to Brueckner et al. (1995) for a description of the LASCO coronagraph on SOHO. Superposed on the LASCO images are the UVCS slit fields of view at the nominal slit heights and orientations.

The plots show that the intersections of the UVCS slit field of view with the borders of the streamer structures, well identified in the image on the top of each figure, are characterized by very sharp, anticorrelated variations of the $\mathrm{O}$ VI line intensity and width, occurring in a PA interval of at most $5^{\circ}-10^{\circ}$. This is particularly evident along the slit (a), at nominal heliocentric distances $\geq 1.75 R_{\odot}$ and $\mathrm{PA} \leq 310^{\circ}$ (Figs. 8-10), when it intersects the boundaries of both streamers appearing in the NW quadrant: we note considerable drops in line intensity (more than one order of magnitude), coinciding with a steep rise in line width (up to a factor of 4), and vice versa. The profile along the slit of the Ly $\alpha$ line intensity, and even more that of its line width, is definitely smoother.

The O VI resonance doublet line ratio also exhibits some interesting features. Along both slit (a) and (b) at $1.51 R_{\odot}$ (Fig. 7), for instance, moving from the denser streamer to regions outside it causes a decrease in the line ratio, from $\sim 0.35$ to $0.25-0.3$. This is the evidence of a significant drop in density, which makes the contribution to the line emission of the resonantly scattered component considerably more important than that of the collisionally excited one (Noci et al. 1987).

Another interesting feature is the steep rise in the O VI intensity line ratio, from $\sim 0.35$ to values $\geq 0.5$, noticed in both slit (a) and (b) at $2.21 R_{\odot}$ (Fig. 10); it occurs at $5^{\circ}-10^{\circ}$ above the Northern border of the low latitude streamer, in a PA interval of $\sim 5^{\circ}$. This is the signature of a fast outflow outside of the streamer above $2.2 R_{\odot}$, because according to Noci et al. (1987) and Dodero et al. (1998) values of the O VI line ratio larger than 0.5 imply radial velocities higher than $100 \mathrm{~km} \mathrm{~s}^{-1}$.

\section{Conclusions}

Our investigation of the streamer-coronal hole interfaces, carried out using a higher latitudinal resolution than previous studies, has shown some interesting features in the behaviour of the examined physical parameters across the streamer boundaries.

We note that both the line intensities, related to the plasma density, and the line widths, related to the kinetic temperatures of the emitting ions, exhibit sharp variations across the streamer boundaries. The steep changes in these quantities occur in a narrow transition region $\left(5^{\circ}-10^{\circ}\right)$, right at the borders of the streamers. The relative variations of the H I Ly $\alpha$ line width, however, are about one order of magnitude smaller than those of the O VI $1032 \AA$ line width, which considerably broadens just outside of the streamer, from $1.5 R_{\odot}$ onwards. Moreover, the variations in O VI line intensity and width are strongly anticorrelated all over the latitudinal intervals considered, whereas those for $\operatorname{Ly} \alpha$, anti-correlated at higher latitudes, also exhibit a positive correlation in the region close to the equator. The different behaviour of the widths of the H I and O VI profiles across the streamer boundaries can be interpreted as a clear evidence of a stronger heating of $\mathrm{O}$ VI ions in the direction coinciding with the line of sight in the open magnetic field region (e.g., Cranmer et al. 1999), assuming that the streamer border defined according to the $1 / e \mathrm{OVI}$ intensity is close to the interface between open and closed field lines (see, also, Abbo \& Antonucci 2002a).

Therefore, the steep broadening of the O VI line widths is an evident signature of the borders of streamers. Thus it may be possible to use these measurements, making latitudinal 

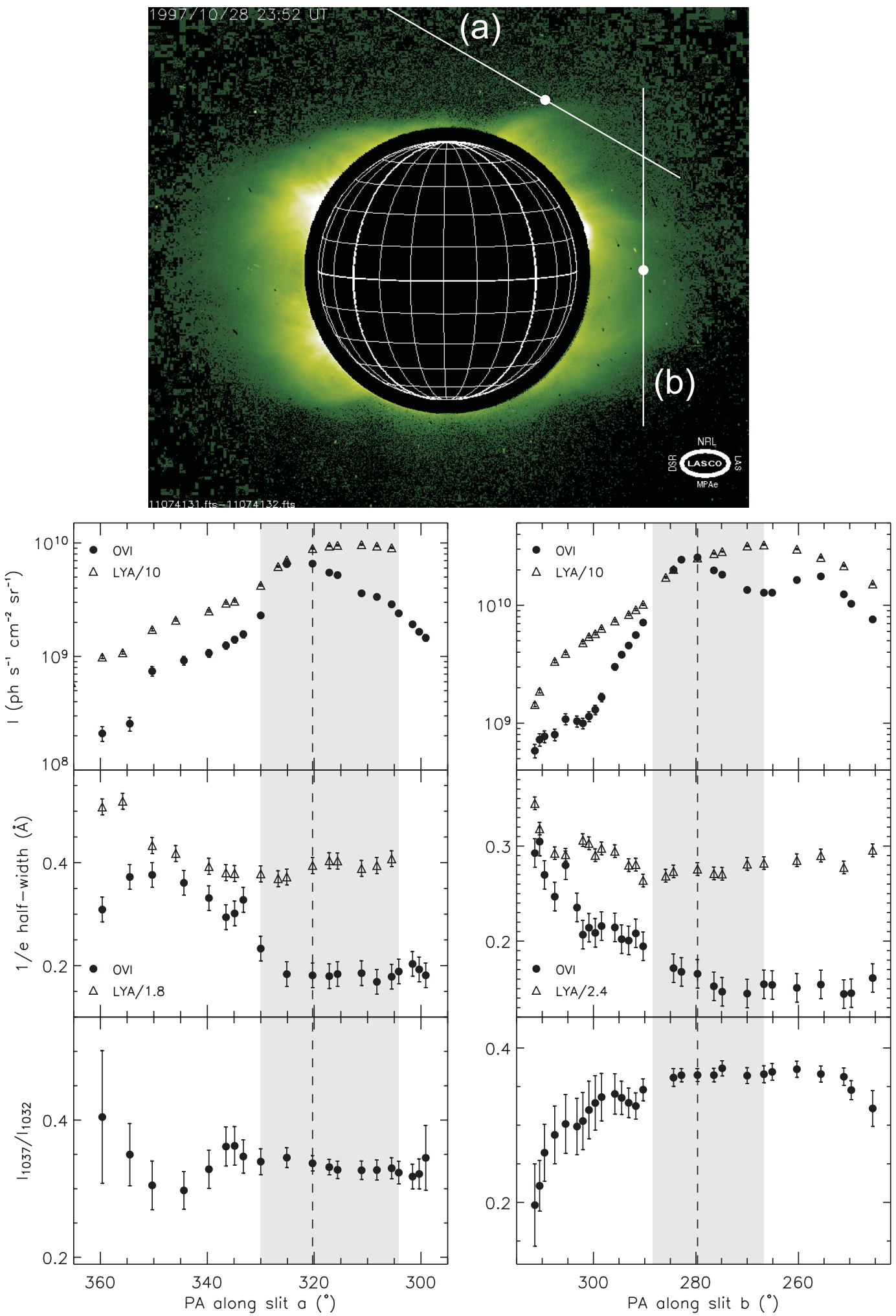

Fig. 7. LASCO C1 image of the solar corona at the centre of the Fe XIV $5303 \AA$ line on October 28, 1997. Superposed on the LASCO image are the UVCS slit fields of view (white lines) at slit height of $1.51 R_{\odot}$, for PA $=330^{\circ}$ (a) and PA $=270^{\circ}(\mathbf{b})$. The nominal height and PA for each slit refer to the distance and PA of the point along the slit closest to the limb (shown as a white dot). The Ly $\alpha$ and O VI $1032 \AA$ line intensities and $1 / e$ half-widths, as well as the $\mathrm{O}$ VI line ratios, vs. position angle along slit $\mathbf{a}$ ) and $\mathbf{b}$ ) are shown in the bottom of the figure. The positions of the streamer axes and borders marked in the panels have been identified as in Fig. 1.

maps at many heights, to provide an accurate indication of the boundaries between the closed and open regions of the streamer magnetic field, as already suggested by Antonucci et al. (1997) and Strachan et al. (2002), and to infer the shape of the closed 
field regions. It is also worth noting, in this respect, the significant reduction in the $\mathrm{O}$ VI line widths occurring in coincidence with the narrow, faint streamer observed at high latitudes $\left(\mathrm{PA} \sim 320^{\circ}\right)$. In this case the streamer morphology can be determined more easily through the characteristics of the line profiles than for the intensity of the lines.

The O VI resonance doublet line ratio, related to the radial outflow velocity, steeply increases outside of the streamer as well, even if this occurs only at heliocentric distances above $2.2 R_{\odot}$. Here we note a sharp transition as a function of latitude from negligible outflows anywhere within the streamer and at its borders to outflow velocities higher than $100 \mathrm{~km} \mathrm{~s}^{-1}$ at $5^{\circ}-10^{\circ}$ from the streamer edges. Closer to the solar limb, conversely, we note a sharp decrease in the line ratio across the streamer boundaries, caused by the density drop in the regions outside of the streamer and, probably, also by an outflow starting along the open magnetic field lines with velocities around 40-50 $\mathrm{km} \mathrm{s}^{-1}$ (see, e.g., Noci et al. 1987; Dodero et al. 1998; Cranmer et al. 1999).

Hence the behaviour of the O VI kinetic temperatures as a function of both heliocentric distance and latitude evidenced by these observations leads us to deduce that the enhanced nonthermal heating of the emitting ions already take place near or just outside of the streamer edges, even close to the solar limb. Such energy deposition increases with distance from the border of the streamer, presumably associated with the opening of the magnetic field lines, causing only a gradual acceleration of the solar wind flow, as shown by the behaviour of the O VI line ratio as a function of the heliolatitude from the streamer border. This moderate latitudinal increase in outflow velocity outside of the streamer causes values $\sim 100 \mathrm{~km} \mathrm{~s}^{-1}$ to be reached along the open field lines running close to its borders farther out in the corona than in coronal holes. For comparison, Cranmer et al. (1999) report outflows velocities $\sim 100 \mathrm{~km} \mathrm{~s}^{-1}$ already at $1.6-1.7 R_{\odot}$ in polar coronal holes during the minimum of solar activity. It is worth noting, on the other hand, that a wind velocity slowly varying from the streamer to the coronal hole, as it appears from the present analysis, can support the hypothesis that the slow wind originates in the regions of open magnetic field lines near the streamer-coronal hole boundaries.

This aspect, as well as the slight increase in $\operatorname{Ly} \alpha$ broadening inside streamers, may have important consequences for realistic theoretical models of streamers and the source regions of the slow solar wind.

Acknowledgements. This work was made possible by the UVCS/SOHO instrument and Flight Operations team. The UVCS is the result of a collaborative effort between NASA and the Agenzia Spaziale Italiana (ASI), with a Swiss participation. D. Spadaro and R. Ventura wish to thank all the staff of the MEDOC center at the Institut d'Astrophysique Spatiale in Orsay - Paris for kind hospitality. The authors also wish to thank R. Suleiman for his help in the preliminary reduction of the UVCS raw data. They thank E. Antonucci for her comments and suggestions, which led to a sounder version of the manuscript. This work was supported in part by the Agenzia Spaziale Italiana (contract ASI I/R/84/02).

\section{References}

Abbo, L., \& Antonucci, E. 2002a, in SOLSPA: The Second Solar Cycle and Space Weather Euroconference, ed. A. Wilson (Noordwijk: ESA), ESA-SP 477, 323

Abbo, L., \& Antonucci, E. 2002b, in SOHO 11 Symposium, From Solar Min to Max: Half a Solar Cycle with SOHO, ed. A. Wilson (Noordwijk: ESA), ESA-SP 508, 477

Antonucci, E., Giordano, S., Benna, C., et al. 1997, in Fifth SOHO Workshop, The Corona and Solar Wind near Minimum Activity, ed. A. Wilson (Noordwijk: ESA), ESA-SP 404, 175

Bemporad, A., Poletto, G., Suess, S. T., et al. 2003, ApJ, 593, 1146

Billings, D. E. 1966, A Guide to the Solar Corona (New York: Academic Press)

Brueckner, G. E., Howard, R. A., Koomen, M. J., et al. 1995, Sol. Phys., 162, 357

Cranmer, S. R., Kohl, J. L., Noci, G., et al. 1999, ApJ, 511, 481

Dodero, M. A., Antonucci, E., Giordano, S., \& Martin, R. 1998, Sol. Phys., 183, 77

Domingo, V., Fleck, B., \& Poland, A. I. 1995, Sol. Phys., 162, 1

Dobrzycka, D., Cranmer, S. R., Panasyuk, A. V., Strachan, L., \& Kohl, J. L. 1999, J. Geophys. Res., 104, 9791

Frazin, R. A., Cranmer, S. R., \& Kohl, J. L. 2003, ApJ, 597, 1145

Gabriel, A. H. 1971, Sol. Phys., 21, 392

Gardner, L. D., Kohl, J. L., Daigneau, P. S., et al. 1996, Proc. SPIE, 2831, 2

Gardner, L. D., Smith, P. L., Kohl, J. L., et al. 2002, in The Radiometric Calibration of SOHO, ed. A. Pauluhn, M. C. E. Huber, \& R. von Steiger (Noordwijk: ESA), ISSI SR-002, 161

Habbal, S. R., Woo, R., Fineschi, S., et al. 1997, ApJ, 489, L103

Kohl, J. L., Esser, R., Gardner, L. D., et al. 1995, Sol. Phys., 162, 313

Kohl, J. L., Noci, G., Antonucci, E., et al. 1997, Sol. Phys., 175, 613

Li, X., Habbal, S. R., Kohl, J. L., \& Noci, G. 1998, ApJ, 501, L133

Low, B. C. 2001, J. Geophys. Res., 106, 25141

Marsch, E. 1997, in Fifth SOHO Workshop, The Corona and Solar Wind near Minimum Activity, ed. A. Wilson (Noordwijk: ESA), ESA-SP 404, 135

Noci, G., Kohl, J. L., \& Withbroe, G. L. 1987, ApJ, 315, 706

Noci, G., Kohl, J. L., Antonucci, E., et al. 1997, in Fifth SOHO Workshop, The Corona and Solar Wind near Minimum Activity, ed. A. Wilson (Noordwijk: ESA), ESA-SP 404, 75

Ofman, L. 2000, Geophys. Res. Lett., 27, 2885

Phillips, J. L., Bame, S. J., Barnes, A., et al. 1995, Geophys. Res. Lett., 22, 3301

Press, W. H., Flannery, B. P., Teukolsky, S. A., \& Vetterling, W. T. 1986, Numerical Recipes. The Art of Scientific Computing (Cambridge University Press)

Priest, E. R. 1987, Solar Magnetohydrodynamics (Dordrecht: Reidel) Raymond, J. C., Kohl, J. L., Noci, G., et al. 1997, Sol. Phys., 175, 645 Romoli, M., Frazin, R. A., Kohl, J. L., et al. 2002, in The Radiometric Calibration of SOHO, ed. A. Pauluhn, M. C. E. Huber, \& R. von Steiger (Noordwijk: ESA), ISSI SR-002, 181

Strachan, L., Panasyuk, A. V., Dobrzycka, D., et al. 2000, J. Geophys. Res., 105, 2345

Strachan, L., Suleiman, R., Panasyuk, A. V., Biesecker, D. A., \& Kohl, J. L. 2002, ApJ, 571, 1008

Ventura, R., Spadaro, D., Cimino, G., \& Romoli, M. 2004, A\&A, in press

Wang, Y.-M. 1994, ApJ, 435, L153

Zangrilli, L., Nicolosi, P., Poletto, G., et al. 1999, A\&A, 342, 592

Zangrilli, L., Poletto, G., Nicolosi, P., Noci, G., \& Romoli, M. 2002, ApJ, 574, 477 
D. Spadaro et al.: The interface between streamers and coronal holes, Online Material p 1

\section{Online Material}


D. Spadaro et al.: The interface between streamers and coronal holes, Online Material p 2

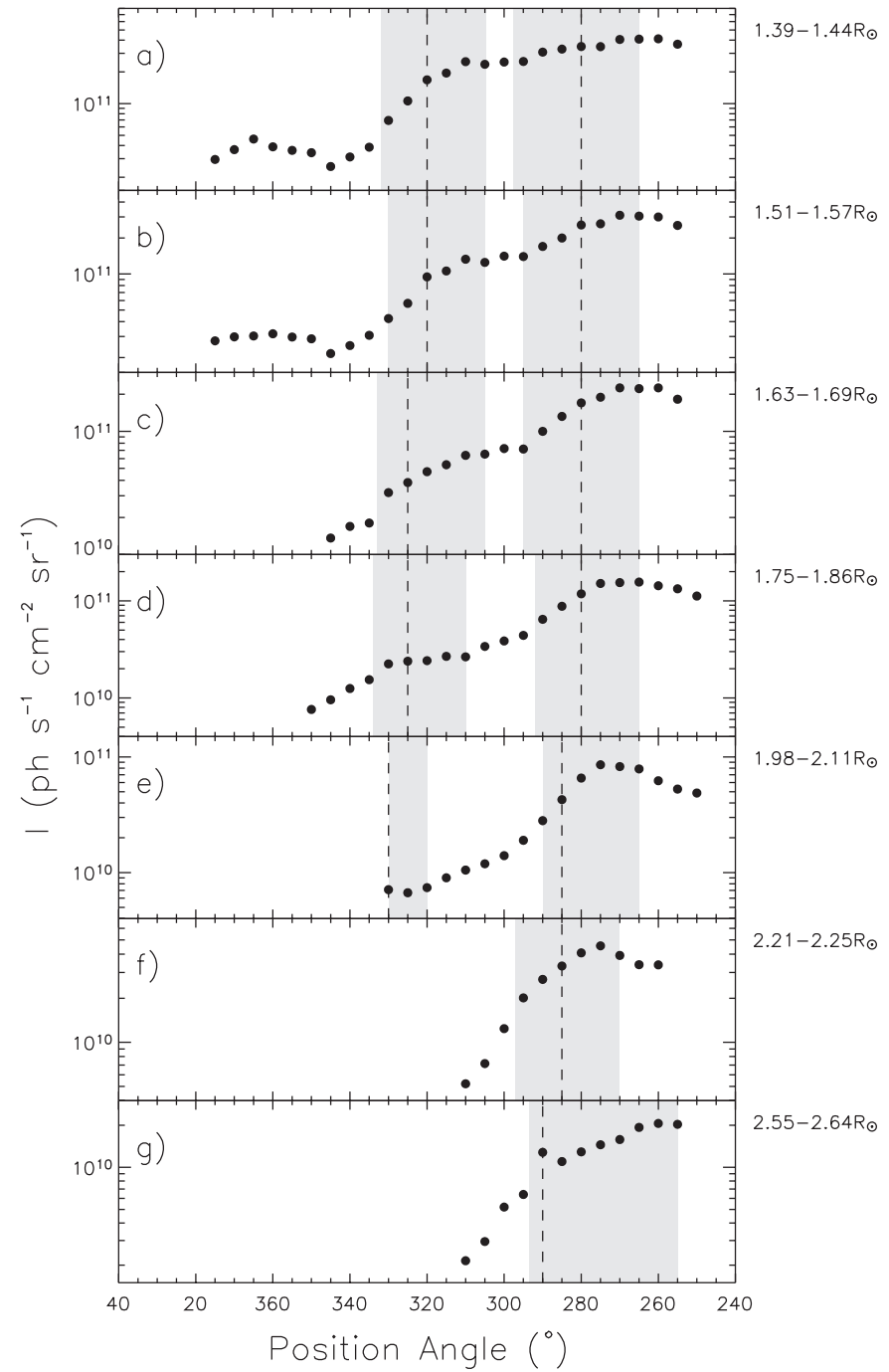

Fig. 2. As in Fig. 1, for the intensity of the H I Ly $\alpha$ spectral line. The marked positions of the axes and borders of the streamers are the same as in Fig. 1, defined according to the behaviour of the O VI line intensity.

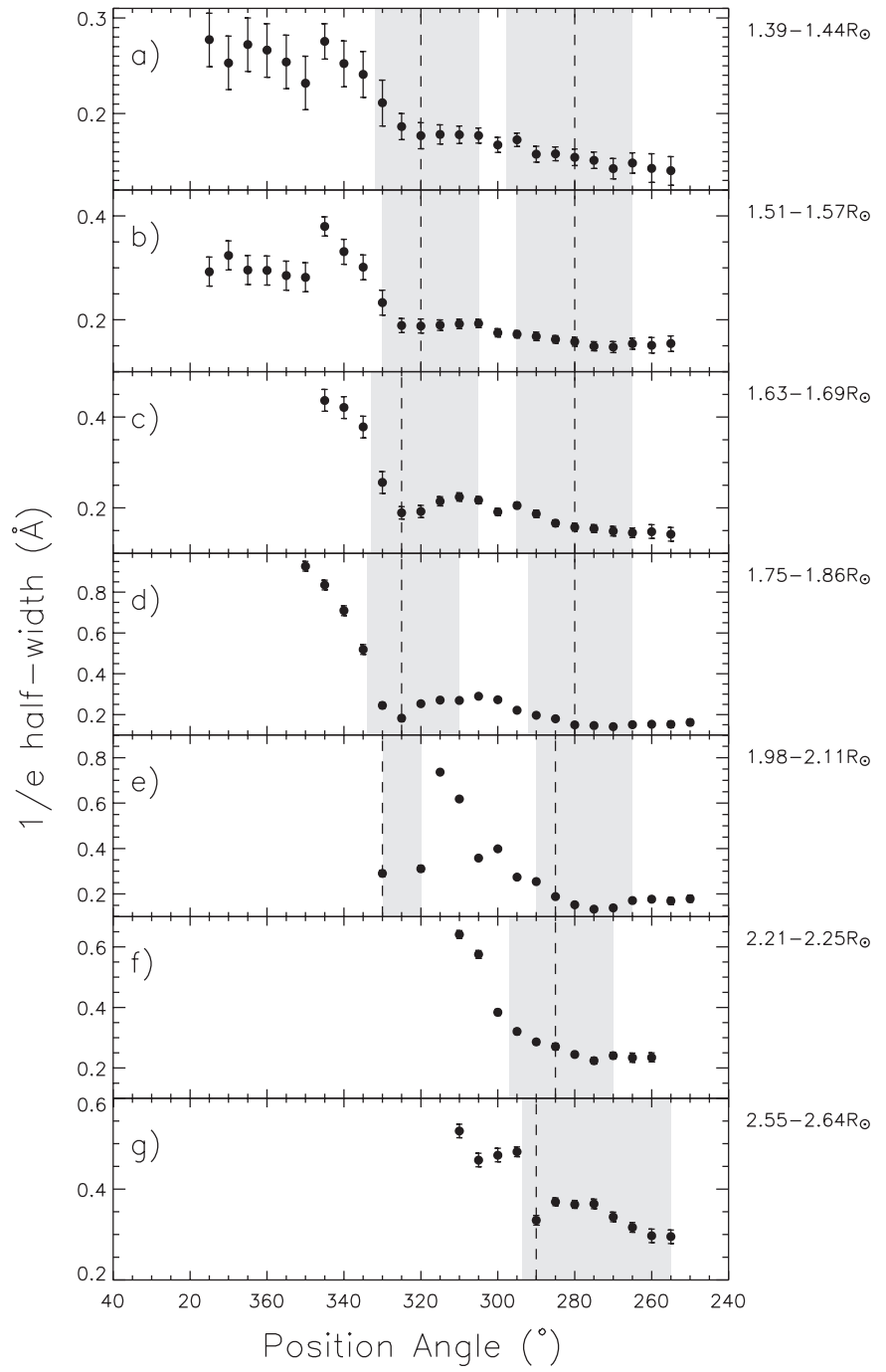

Fig. 4. As in Fig. 1, for the 1/e half-width of the O VI $1032 \AA$ spectral line. 
D. Spadaro et al.: The interface between streamers and coronal holes, Online Material $p 3$

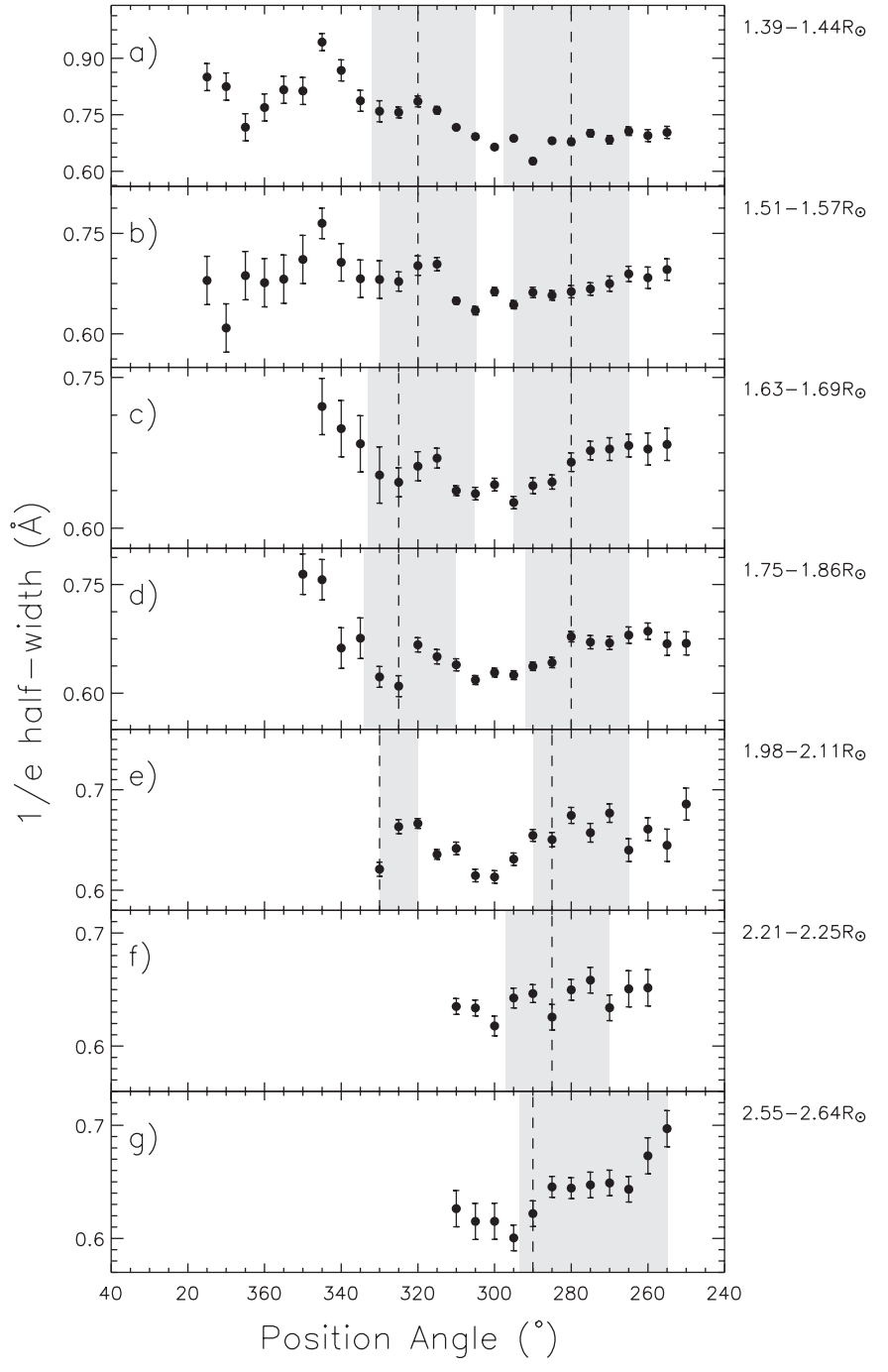

Fig. 5. As in Fig. 2, for the 1/e half-width of the H I Ly $\alpha$ spectral line.

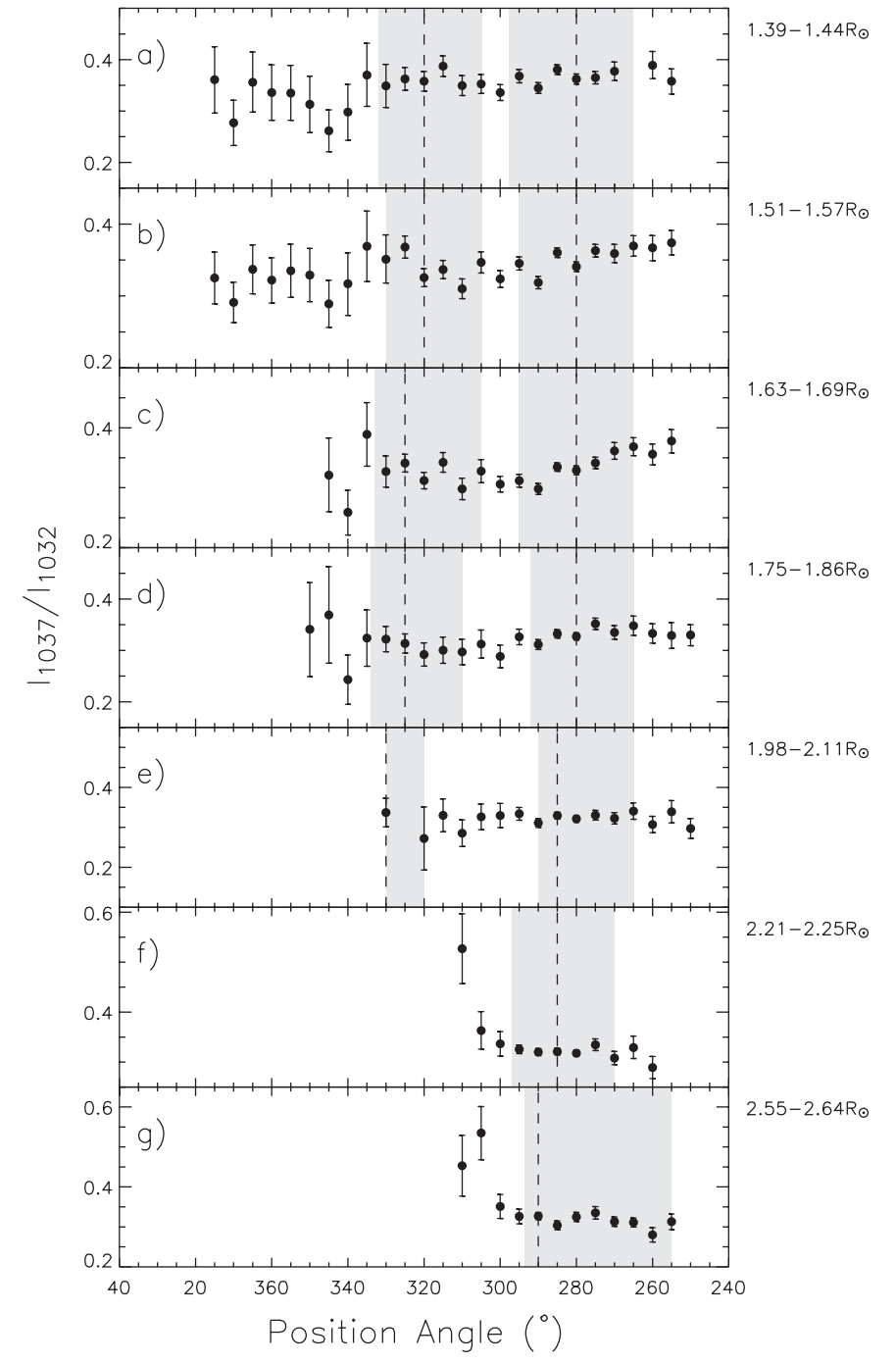

Fig. 6. As in Fig. 1, for the O VI $I_{1037} / I_{1032}$ line ratio. 
D. Spadaro et al.: The interface between streamers and coronal holes, Online Material p 4
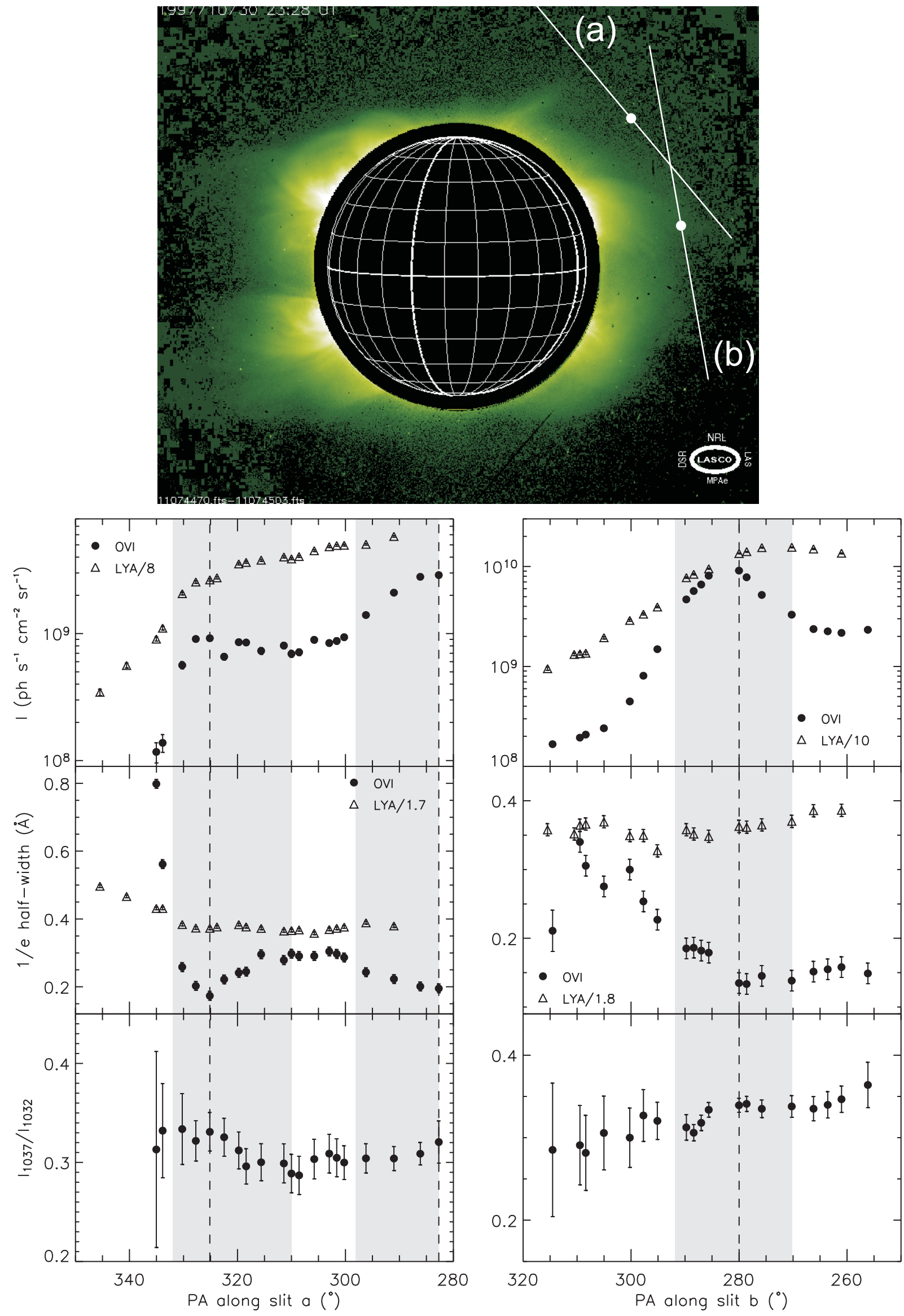

Fig. 8. As in Fig. 7, on October 30, 1997. The UVCS nominal slit height is $1.75 R_{\odot}$, for PA $=310^{\circ}$ a) and $\mathrm{PA}=280^{\circ} \mathbf{b}$ ). 
D. Spadaro et al.: The interface between streamers and coronal holes, Online Material p 5
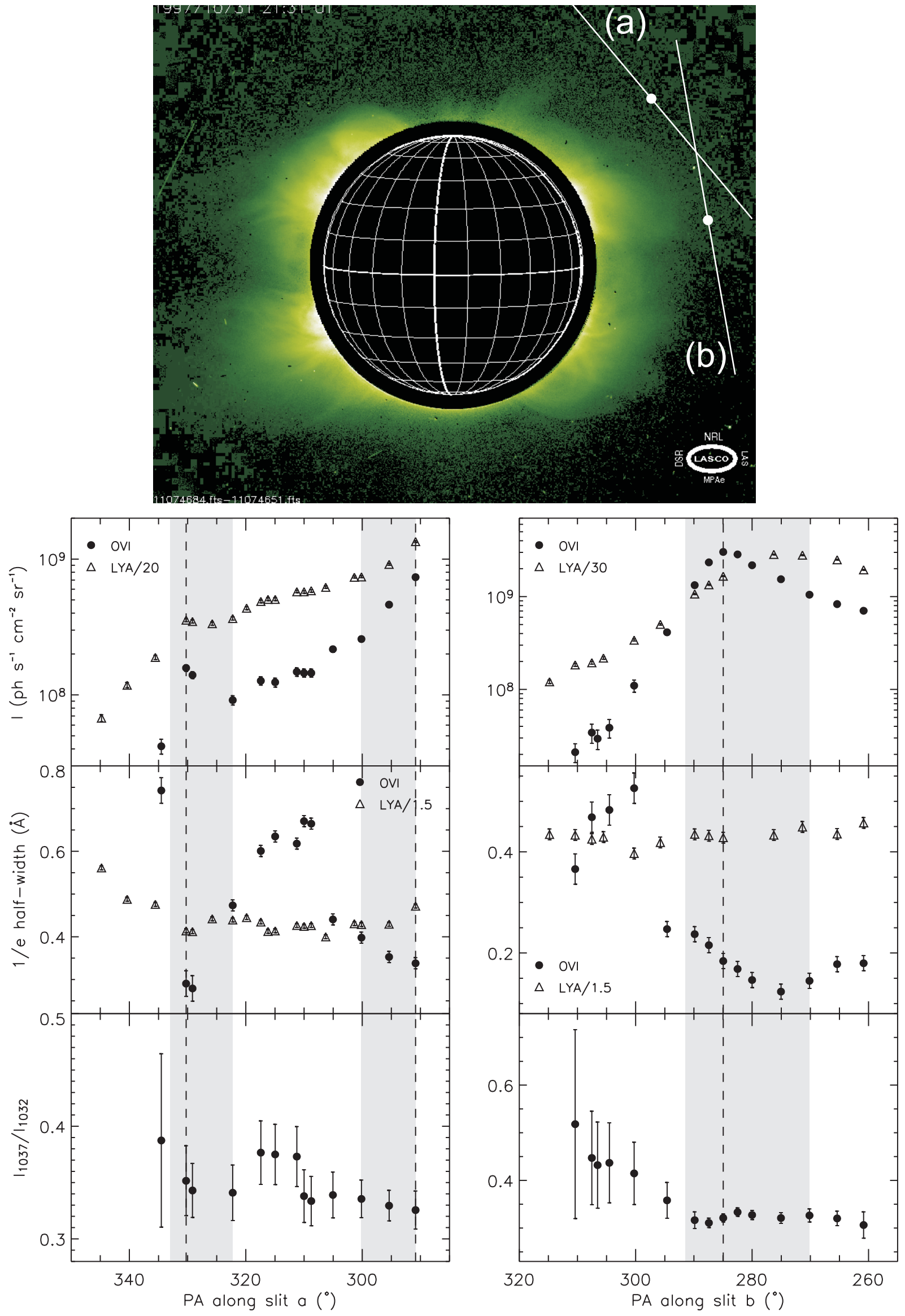

Fig. 9. As in Fig. 7, on October 31, 1997. The UVCS nominal slit height is $1.98 R_{\odot}$, for PA $=310^{\circ}$ a) and $\mathrm{PA}=280^{\circ} \mathbf{b}$ ). 
D. Spadaro et al.: The interface between streamers and coronal holes, Online Material $p 6$
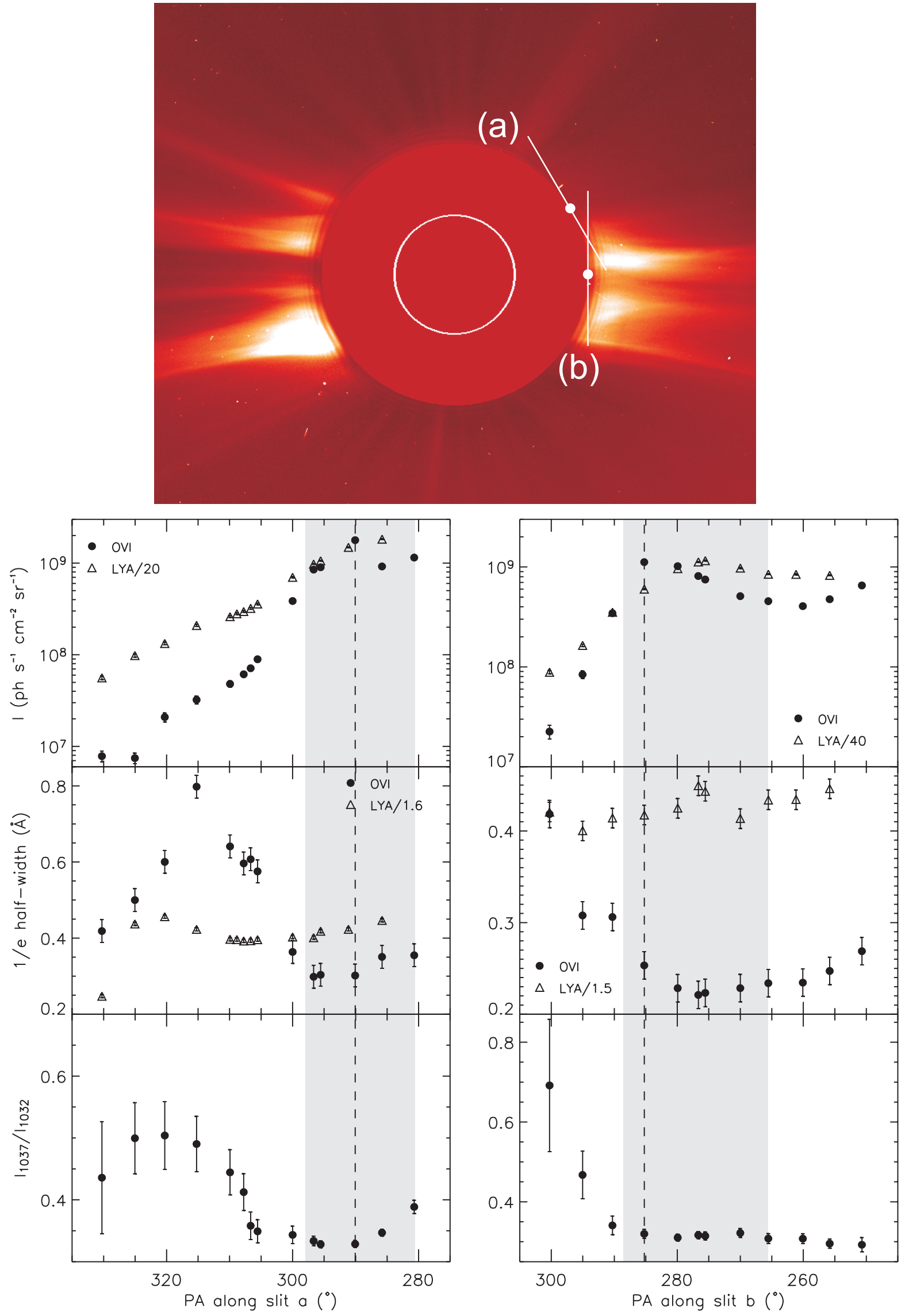

Fig. 10. As in Fig. 7, on November 1, 1997, with the image of the extended solar corona obtained by the LASCO C2 coronagraph in broad-band visible light. The UVCS nominal slit height is $2.21 R_{\odot}$, for $\mathrm{PA}=300^{\circ} \mathbf{a}$ ) and $\mathrm{PA}=270^{\circ} \mathbf{b}$ ). 